\title{
Mother-Child Conversation and Children's Understanding of Biological and Nonbiological Changes in Size
}

Jennifer L. Jipson and Maureen A. Callanan

Jennifer L. Jipson and Maureen A. Callanan, Department of Psychology, University of California, Santa Cruz.

This research was supported in part by funds from a National Institute of Child Health and Human Development grant (HD 26228) and funds from University of California, Santa Cruz. We thank Nameera Akhtar, Susan Gelman, Mark Sabbagh, and two anonymous reviewers for helpful comments. We also thank Molly Fuller, Erin McClure, and Michelle Bialon for assistance with data collection and coding, and Community Children's Center, Neighborhood Child Care Center, and A Child's Reflection for their participation. Portions of this work were presented at the 1995 and 1997 biennial meetings of the Society for Research in Child Development, and at the 1996 and 1998 biennial meetings of the Southwest Society for Research in Human Development.

Correspondence concerning this article should be addressed to Jennifer L. Jipson, Psychology Department, University of Michigan, Ann Arbor, MI 48109. Electronic mail may be sent to jiipson@umich.edu.

This article explores the ways that mothers and children from primarily middle-income European American backgrounds reason about events in which biological and nonbiological objects change in size. In Study 1, mother-child conversations were examined to investigate the events mothers described as growth, as well as the ways mothers explained events occurring in different domains. Findings indicate that although mothers primarily discussed events in domain-specific ways, they exhibited some 
domain blurring in their talk to children. In Study 2,3-year-old children ( $M=3$ years, 2 months) and 5-year-old children $(\mathrm{M}=5$ years $)$ provided descriptions and explanations of the same events. Results suggest that preschool children have begun to develop domain-specific understandings. Results are discussed in light of the role that social interaction plays in children's conceptual development.

With the purpose of characterizing early thought, many researchers have closely examined children's understanding of core areas of knowledge identified by adults, such as psychology (e.g., Gopnik \& Wellman, 1994), physics (e.g., Carey \& Spelke, 1994), and biology (e.g., Hatano \& Inagaki, 1994). This body of work has yielded valuable information regarding what children know about particular objects and events and when this knowledge becomes differentiated by domain. Largely unanswered, however, is the question of how children come to their domain-specific understandings (Coley, 2000; Gauvain, 2001; Wellman \& Gelman, 1998).

In this article we focus on social interaction as a potential mechanism of conceptual development. As children attempt to make sense of their world, they are likely to do so in the context of social activity. Although domain distinctions in everyday phenomena may be readily apparent to adults, an open question is whether and how these differences come to be noticed by children. One possibility is that adults might differentiate by domain in the ways they talk with children about a variety of events. It is also possible, however, that adults may not consistently talk about events in domain-specific ways.

As an example, consider the multiple ways the concept of growth is used in our culture. In addition to the biological sense of the word grow, ("the flowers are growing"), children might hear people say that "the economy is growing," or they may see crystal-growing kits in their local toy store. And while reading Lewis Carroll's Alice's Adventures in Wonderland, they 
may notice that the caterpillar tells Alice, "One side will make you grow taller, and the other will make you grow shorter." It is clear from these examples that the English language allows for flexible uses of certain concepts and, as Keil (1994) pointed out, "true definitions are rare occurrences in word meanings." Thus, although growth is perhaps most commonly thought of as a critical biological process, there are many other possible applications of the concept. Little is known about the extent to which children encounter semantic ambiguity in their interactions with others, or whether such ambiguity influences children's conceptual development.

In the present studies we focus on preschool children's developing theories with two primary goals. First, we explore mother-child conversations as a context within which children may have opportunities to distinguish biology as a separate domain. Second, we examine children's explanations of real-world events that are structurally equivalent in some surface respects yet fall into different domains (biological, nonbiological natural, artifact).

The ability to distinguish biological from non-biological kinds has been central to recent debates over whether young children possess intuitive biological theories distinct from their theories of other domains. Past work has shown that children as young as 3 years of age distinguish between living kinds and artifacts in the causes they consider appropriate to explain events (Gelman \& Gottfried, 1996; Hatano \& Inagaki,1994; Springer \& Keil,1991). Children are particularly adept at making the living kind-artifact distinction when asked about familiar biological properties such as eating and growth (Inagaki \& Hatano, 1996).

In this article we focus on how mothers and children invoke the concept of growth, as this concept seems to be central to children's emerging biological theories (Inagaki \& Hatano, 1996). To date, studies exploring children's understanding of growth have focused on their judgments of the potential for animals, plants, and human-made artifacts to grow. Rosengren, Gelman, Kalish, and McCormick (1991), for example, demonstrated that children as young as 3 years of age knew that animals grow and that nonliving things do not grow. Inagaki 
\& Hatano (1996) extended this work to a broader range of living kinds and found that 4-yearold children also attribute growth to plants (see also Backsheider, Shatz, \& Gelman, 1993). This finding suggests that children's reasoning about the potential for growth centers around whether an object is alive rather than whether it is animate.

Other researchers have found that children not only honor domain distinctions in their judgments about growth but they also know something about the processes involved in growth. Inagaki \& Hatano (1987) found that 5-year-olds understand that living things do not grow because one wants them to (i.e., by human desire) but grow because of specific biological processes (e.g., eating, getting water). Also, Hickling and Gelman (1995) demonstrated that by 4 years of age children understand that seeds grow because of certain natural mechanisms (e.g., rain, sunshine). These studies suggest that growth seems to be a concept that preschool children know a lot about, leading some researchers to suggest that growth "may constitute the core of young children's concept of living things" (Inagaki \& Hatano, 1996, p. 2825).

The existing work, however, fails to explore fully the extent of children's knowledge about growth for several reasons. First, many of these studies relied on forced-choice tasks that may overestimate children's tendency to differentiate by domain in the types of explanatory processes they use. For example, Backsheider et al. (1993) asked children to judge whether damaged animals, plants, and artifacts could heal through regrowth or whether a person could repair them. Children differentiated by domain in the causes they selected, attributing the possibility for regrowth only to plants and animals. It is not clear from these findings, however, whether children would differentiate by domain as clearly if asked to generate their own explanations for these events.

A second problem with current work lies in the items selected by many researchers to investigate domain differences in children's judgments of growth. Rosengren et al. (1991), for example, compared questions about whether animals get bigger over time with 
questions about whether artifacts such as chairs and teapots increase in size over time.

Although preschool children easily distinguished between these events, it may be premature to draw the conclusion that children see growth as a biological concept without also investigating children's reasoning about artifacts that actually do get bigger. In their everyday lives children are likely to encounter events in which nonbiological objects increase in size (e.g., a beach ball being blown up). It is not known whether children would judge actual increases in size of artifacts as growth.

Another shortcoming of current work, also related to item selection, is that it has focused exclusively on children's understanding of whether biological kinds and artifacts grow, excluding non-biological natural kinds (e.g., Inagaki \& Hatano, 1996; Rosengren et al., 1991). The distinction between biological and nonbiological natural kinds has theoretical importance. Both are natural objects; however, only biological kinds are living. Current work has often focused on the important conceptual distinction between biological (living) kinds and nonliving kinds, but the nonliving kinds studied have been primarily human-made artifacts. In one exception, Gelman and Kremer (1991) explored the distinction between natural kinds and human-made artifacts, showing that 4-year-old children differentiate natural objects from human-made objects in terms of their explanations of origins. In this study, however, living and nonliving natural kinds were grouped together. Little is known about whether children understand changes in size in different ways depending on whether the object undergoing change is a living thing, a nonliving natural-kind object, or an artifact.

In the present research we aim to clarify the preceding issues by exploring participants' explanations of and talk about events in which objects from three ontological categories change in size (biological kinds, nonbiological natural kinds, and human-made artifacts). In addition, the use of an open-ended task encourages participants to talk about depicted events in an 
unconstrained manner. Thus, both the design and the methodology of this study will broaden our knowledge of children's understanding of real-world events.

In addition to contributing to knowledge about children's domain-specific understandings, we also investigate how children come to understand the world in domain-specific ways. The process by which children advance their understandings is often discussed in terms of children's active attempts to make sense of the world through self-directed exploration and experimentation (e.g., Carey \& Spelke, 1994; Gopnik \& Meltzoff, 1997). Recent sociocultural views, in contrast, have emphasized the significance of the social and cultural contexts within which children negotiate their understandings (e.g., Rogoff, 1990). In particular, conversations with family members may be an important context in which young children begin to develop and refine their intuitive understandings.

Despite increasing recognition of the value of studies that examine family discourse as it relates to theory development (Inagaki \& Hatano, 1996; Wellman \& Gelman, 1998), very little work has focused on the content of parent speech to children as it relates to children's developing theories of biology (in contrast to work on mother-child conversation and children's theory-of-mind development; e.g., Bartsch \& Wellman, 1995; Ruffman, Slade, \& Crowe, 2002; Sabbagh \& Callanan, 1998). One exception is a study by Gelman, Coley, Rosengren, Hartman, and Pappas (1998) in which mother-child dyads were asked to read a picture book designed to elicit talk about animal and artifact categories. Findings revealed that mothers tended to stick to labeling objects and providing generic category information (e.g., " dogs bark'), rarely offering causal explanations to their children. Several other studies suggest, however, that important conversations about causality may be more prevalent outside of bookreading settings (Beals, 1993; Crowley et al., 2001; Snow \& Kurland, 1996). Such conversations may support intuitive theory development, particularly if parents reserve certain types of explanations for events occurring in certain domains. In this study, we examine whether 
parents, in conversation with their children, talk in ways that might help children recognize the domain-specific nature of certain causal processes.

In sum, the present studies contribute to the literature on parent-child conversations, as well as to the literature on children's biological understanding. In Study 1, we provide information about how mothers discuss biological and nonbiological events with their children. In Study 2, we present new evidence regarding children's understanding of growth and their explanations of biological and nonbiological changes in size. The results of this research add to our understanding of what children know and shed light on how social interactions may contribute to children's emerging theories.

Study 1

In our first study, we examine the ways mothers talk with children about various increases and decreases in size to gain insight into the potential role mothers may play in children's developing understanding of biological and nonbiological phenomena. Because prior work has demonstrated that preschool children attribute growth to living but not nonliving objects, we pay particular attention to whether mothers of preschool children talk about growth strictly for biological changes, or whether they demonstrate flexibility in the changes they identify as growth. In addition, we look at the explanations mothers provide for events occurring in different domains.

\section{Method}

\section{Participants}

Thirty-six mother-child pairs participated. The sample was divided evenly into two groups based on child's age: 3 -year-olds $(\mathrm{M}=3$ years, 2 months; range $=2$ years, 7 months to 3 years, 11 months) and $4{ }^{1}{ }_{2}$-year-olds $(\mathrm{M}=4$ years, 7 months; range $=4$ years, 1 month to 4 years, 11 months $)$. 
There were an equal number of boys and girls in each group. Mothers were mainly from middle-income European American backgrounds and all were native English speakers. Participants were recruited through local child care centers in a coastal region of Northern California; they participated voluntarily and were given a children's book as a thank-you gift.

\section{Materials}

Ten picture sets depicting physical change-of state events were used. Each set consisted of a series of three pictures in sequence, for example, a deflated beachball, a slightly more full beachball, and a blown-up beachball. Eight of the items depicted differed by domain (biological, artifact) and direction of change (increasing, decreasing). Thus, two items depicted biological increases, two depicted biological decreases, two depicted artifact increases, and two depicted artifact decreases. We included two additional events depicting the actual and apparent increase in size of natural-kind objects that are not alive (these items are henceforth referred to as natural-kind items). Within each category, we deliberately selected an assortment of items to explore the variety of contexts within which children might hear talk about growth and a range of causal mechanisms. By doing so we test the scope of language use by asking parents to discuss picture sets ranging from the depiction of prototypical biological growth (e.g., a duck growing) to picture sets depicting apparent, but not actual, change in size of nonliving natural-kind items (the waxing of the moon). For specific items see Table 1.

Pictures used in 6 of the 10 picture sets were taken from various children's books; pictures used in the remaining 4 sets were photographs taken by the researcher. All picture sets were presented in a picture book entitled, What Happened to the

\section{Procedure}


All sessions were held in a university laboratory playroom. Upon arriving at the playroom, mothers were provided with information regarding the procedure and were asked for informed consent to participate. After a brief free-play period, the picture book activity was introduced. Mothers were told that the researchers were interested in how young children and their mothers talk about objects and events in the world and were asked to look through the picture book with their child in whatever way seemed natural to them. The researcher then left the room for the duration of the mother-child session and videotaped their interaction from an adjacent room. The average session length was approximately $15 \mathrm{~min}$ (range $=9 \min$ to 28 $\min )$.

\section{Coding}

Reference to growth. Using transcripts and videotapes of the sessions, we first identified every instance in which mothers used the word grow (or a variation). We then coded whether each instance was an actual description of the depicted event. This step was necessary because we found that mothers could talk about growth in several ways. First, mothers could actually describe an event as a growth event (e.g., "The mushroom grew taller"). Second, they could talk about growth as being a precursor or antecedent to a depicted event without actually describing the depicted change as growth (e.g., in the series of pictures showing a strawberry rotting, "The strawberry grew and grew and then it didn't get picked so it rotted"). Third, they could mention growth in the form of a question (e.g., "Did the mushroom grow?"). All instances in which mothers used the word grow (or a variation) were coded by two independent coders according to whether the term was being used as an actual description of the depicted change. Agreement on this judgment between two independent coders was $86 \%$. For cases in which coders disagreed as to the use of grow, coders jointly reviewed the transcript and videotape and came to a mutual conclusion. This tended to be fairly straightforward as disagreements were 
often due to one coder noting an aspect of the utterance overlooked by the other coder, such as intonation (e.g., "It grew” versus “It grew?”). In conducting our analyses, we focused exclusively on cases in which mothers were judged to have actually described the depicted event as growth.

\section{[Insert Table 1]}

Explanations. Mothers' explanations of picture sets were coded to explore whether they explained events occurring in different domains in distinct ways. Explanations were coded according to the following categories: (a) organic cause: explanations in this category involve the mention of processes or factors that adults recognize to be important in biological growth, such as food (e.g., "He grew because he ate a lot of food"), water (e.g., "He drank a lot of water"), origins (e.g., "It grew from a tiny seed”), and natural environment (e.g., "Mushrooms need cool dirt to grow"); (b) human cause: explanations in this category were coded when an event was said to be due to human action (e.g., "Someone must have blown it up"); and (c) other: explanations in this category included any stated cause for an event, apart from organic and human cause, such as the passing of time (e.g., "After it sits for a while it gets smaller"), intentions (e.g., "The puffer fish decided to go back to its usual size”), and purpose (e.g., "It grew big so we could eat it"). In addition to these explanation codes, we also coded no explanation for cases in which no causal mechanisms were suggested for the event. We expected mothers to explain biological events with organic cause explanations and artifact events with human cause explanations. No predictions based on domain were made for other explanations.

These codes were not mutually exclusive; for example, a participant could explain the growth of the mushroom by saying, "Someone gave it water." This example would be coded as both organic and human cause. Intercoder reliability was established by two coders independently coding $25 \%$ of the transcripts. Using Cohen's kappa as the agreement statistic, 
reliability for this decision was 0.71 (percent agreement $=78 \%$ ); again, most disagreements involved one coder missing something, and all disagreements were readily resolved through discussion. This kappa indicates a good level of agreement (Bakeman \& Gottman, 1997).

\section{Results and Discussion}

\section{Mothers' Reference to Growth}

One research question asked whether mothers mainly referred to certain kinds of changes as growth (e.g., increases only, biological changes only) or whether they attributed growth to a variety of changes. Inspection of mothers' talk about growth revealed a clear effect of direction in that mothers used the word grow exclusively to refer to events depicting increases in size. To explore the effects of domain and age, a 2 (age: 3 years, 41 years) x 3 (domain: biological, artifact, natural kind) mixed ANOVA was conducted on the number of picture sets per event type that mothers discussed as growth. Each mother received three scores, one for each type of event (biological increases, artifact increases, and natural-kind increases). These scores could range from 0 (mother did not describe either of the picture sets within a category as growth) to 2 (mother described both picture sets within a category as growth).

Although the number of zeros in the data set raised concerns about homogeneity of variance, Box's M test was not significant, therefore indicating stable spread, $F(6,8,375)=1.90$, $p$

5.36 . Results revealed a main effect of domain, $\mathrm{F}(2,33)=52.16, \mathrm{p}<.001$, with Tukey's HSD post hoc analyses indicating that mothers talked about growth more often when describing biological events than when discussing either artifact or natural-kind events, $\mathrm{p}<.05$ (see Table 2 for means). In addition, mothers talked about growth more for natural-kind events than for artifact events (HSD, $\mathrm{p}<.05$ ). This finding seems to be driven by the tendency of many mothers to refer to crystal accretion as growth. 


\section{[Insert Table 2]}

These comparisons suggest that although mothers tend to concentrate on biological increases in size when talking to their young children about growth, they do sometimes discuss nonbiological events in terms of growth. Although this study does not speak to whether such nonbiological uses influence children's conceptual understandings, inconsistency in maternal talk to children may complicate the learning process. Next, we look descriptively at the conversations surrounding mothers' nonbiological uses of grow to answer the following question: When mothers talk about growth for nonbiological events, do they indicate this may be a different application of the term? If so, it is possible that rather than complicating the learning process, flexibility in word use might actually be packaged in a context that provides information valuable to conceptual development?

To address this issue, mothers' description of nonbiological events as growth events were coded to determine whether they indicated they were using grow in an unusual way. Percent agreement on this decision by two independent coders was $100 \%$. Results indicate that on $44 \%$ ( 8 of 18) of the nonbiological items that mothers discussed as growth, mothers qualified their use of the term in their description. It is interesting that all qualified uses of grow were for the crystal, suggesting that, for mothers, this event highlighted the conflict between everyday language use (in which crystals are often talked about as growing) and the desire to help their children understand growth as a biological process. In an example of this kind of conversation, one mother explained crystal formation by saying, "It's kind of a rock that kinda grows. It doesn't really grow because it's not alive, but it grows because it adds more and more of the rock to it." This mother not only qualified her use of the word grow but also provided information about the ontology of crystals ("a kind of rock") and suggested something about the domain specificity of the process of growth (as related to living kinds). Furthermore, this mother implicitly introduced the possibility that there may be multiple ways to think about 
the concept of growth, beyond its strict biological meaning. In a similar example, after a mother told her child that crystals grow she went on to say "but they're not alive but they grow anyway." It will be interesting in future work to test directly whether talking about how nonbiological appeals to growth deviate from biological uses helps children have a clearer understanding of biological events.

\section{Mothers' Explanations}

Biological and artifact items. To explore patterns in mothers' use of various causal mechanisms to describe biological and artifact events, mothers' explanation scores were submitted to a MANOVA as dependent variables. In this MANOVA, child age was a betweensubjects factor, and explanation type, domain, and direction were within-subjects factors. Analyses were based on the number of picture sets per event type (biological increases, biological decreases, artifact increases, and artifact decreases) for which mothers used each of the three explanation categories to explain the depicted change. Mothers' mean use of each causal explanation is shown in Table 3.

Inspection of the means and standard deviations in Table 3 raised concerns about constrained variance; as a result, the MANOVA was repeated with the data transformed using a natural logarithmic transformation (as suggested by Emerson \& Soto, 1983). The results from the analysis using transformed data replicated the results reported here. The MANOVA revealed a significant main effect of domain, $\mathrm{F}(1,34)=26.44, \mathrm{p}<.001$, with mothers' offering explanations for more artifact events $(M=3.97)$ than biological events $(M=1.89)$. In addition, a main effect of explanation type was found, $F(2,33)=13.93, p<.001$. Tukey's post hoc analyses demonstrated that mothers used other explanations more than either organic or human cause explanations, and human cause explanations more than organic cause explanations, $\mathrm{p}<.05$. 


\section{[Insert Table 3]}

Several interactions were also revealed by the analysis. First, explanation type and direction interacted, $\mathrm{F}(2,33)=29.30, \mathrm{p}<.001$. Tukey's HSD post hoc analyses indicated that mothers used organic cause explanations more for increasing events than for decreasing events; however, the pattern was reversed for mothers' use of other explanations, $p<.05$. Mothers used human cause explanations equally for increasing and decreasing events. Domain and direction also interacted, $\mathrm{F}(2,33)=5.31, \mathrm{p}<.05$. When talking about biological changes in size, mothers explained more decreasing than increasing events $(\mathrm{HSD}, \mathrm{p}<.05)$. There was no effect of direction of change on mothers' explanations of artifact events.

Finally, and most important, explanation type interacted with domain, $F(2,33) 519.97$, p $<.001$. When explaining biological events, mothers used organic cause explanations more than human cause explanations (HSD, $\mathrm{p}<.05)$. For artifact events, mothers used human cause explanations more than organic cause explanations (HSD, $\mathrm{p}<.05$ ). It is interesting that for both biological and artifact events, mothers used other explanations more than either of the other explanation types (HSD, $\mathrm{p}<.05)$. The frequent use of other explanations by mothers seemed to center on their tendency to explain decreasing events with appropriate explanations, such as the passing of time for the strawberry and the loss of air for the balloon.

Natural-kind items. In only nine cases did mothers offer any explanation of a natural-kind event. Of these, two were instances in which a mother used an organic cause explanation (once each for the crystal and moon). In the remaining seven cases, mothers offered other explanations. The following example of a mother talking with her 5-year-old daughter is one of the few cases in which an explanation was offered:

Mother: What happened to the crystal?

Child: First somebody watered it and it grew 
and it grew

Mother: Oh, but a crystal. I don't think it grew on

the ground even though it looks green. But they say it's a crystal so it probably grew underground or maybe inside of a rock. It started out with certain chemicals in certain combinations like this, and then maybe there was pressure from the top and in certain conditions if the chemicals are just right it might start growing like a living thing over time.

In this example, the mother guides her child away from thinking of this as a biological event (akin to plant growth) and suggests an explanation more appropriate to the domain (chemical reactions). This type of interaction suggests that mothers sometimes talk with children in ways that might be helping to support their developing understanding of biology as a separate domain with distinct causal mechanisms. Such conversations, however, were relatively rare. Instead of explaining natural-kind events, mothers often simply provided brief descriptions (e.g., "It's getting bigger"). This may be because the two natural-kind events included in the book depicted relatively complex changes (the waxing of the moon, crystal accretion) that mothers may not have felt their children would understand or may not have fully understood themselves.

\section{Summary of Study 1 Findings}

We found that by attending to the ways mothers talk about a variety of events, children have opportunities to learn about ontological borders and domain-specific causal mechanisms. Mothers talked about growth exclusively for events in which an object increased in size, with an emphasis on biological events. In addition, mothers differentiated between biological and artifact events in their use of organic cause and human cause explanations to 
explain events. Furthermore, mothers refrained from using either type of explanation when discussing natural-kind events.

At times, however, mothers did demonstrate domain blurring in their talk to children. Close inspection of these cases suggests that mothers often did so in appropriate ways. For example, when growth was mentioned outside of the biological context, it tended to be in reference to the crystal, a nonbiological case in which increasing size is often described as "growth" in adult speech. In addition, when mothers used human cause explanations to discuss

biological events, they did so exclusively for either the mushroom or the strawberry (e.g., "They didn't give it enough water so it died"). Similarly, the only biological explanation mothers used to explain artifact events was in reference to the sponge expanding because of water. Thus, in general, mothers differentiated by type of event in the ways they described and explained events, and when they deviated from domain specificity they did so in sensible ways.

\section{Study 2}

In our second study, we examined preschool children's open-ended discussions of common events in which a variety of objects change in size with the goal of investigating whether and how children distinguish by domain in their reasoning about events. We focused on (a) which events children described as growth, and (b) whether children differentiated by domain in the specific causal mechanisms they used to explain change. An important aspect of this work is its use of an open-ended questioning strategy, as well as its inclusion of a varied set of items (including actual artifact increases and natural-kind events). Thus, this project extends previous work and advances our understanding of children's emerging biological knowledge. This research strategy also allows us to look at the ways children discuss events from different domains in light of the way the same events were discussed by mothers in Study 1. 


\section{Method}

\section{Participants}

Thirty-two children from three child care centers located in a coastal region of central California participated. The sample was evenly divided into two age groups: a 3-year-old group $(\mathrm{M}=3$ years, 2 months; range $=2$ years, 8 months to 4 years, 1 month $)$ and a 5 -year-old group $(\mathrm{M}=5$ years, 0 months; range $=4$ years, 5 months to 5 years, 9 months $)$. Each age group consisted of an equal number of boys and girls. The majority of the children were from middle-income, European American backgrounds.

\section{Materials}

Fifteen picture sets depicting physical change-of state events were used. As in Study 1, each picture set consisted of a sequence of three pictures depicting a physical change-of-state event. Twelve of the picture sets showed sequences of increases and decreases in size across two domains: biological kinds and artifacts. Three additional picture sets depicting the actual and apparent increase in size of nonliving natural-kind objects were also included. (Note that whereas in Study 1 mothers saw two events of each type, children in this study saw three events.) See Table 1 for specific items.

\section{Procedure}

All sessions were conducted by the same researcher in a quiet area of each preschool and were audiotaped. Each child participated individually in a single session of testing. Children viewed all 15 picture sets in one of three random orders, with the only constraint on the orders being that no more than two picture sets of the same type (e.g., biological increase) could appear consecutively. 
While pointing to the pictures one at a time, the researcher said: "Here is a picture of a (object name),"' then, "Here is another picture of the (object name) that was taken later on,'” and finally, "Here is one last picture of that (object name) that was taken even later on." Although the majority of the picture sets depicted changes of state of common items, object labels were provided to ensure that children knew what the pictures represented. Furthermore, for all items the phrases later on and even later on were given vocal emphasis to accentuate the idea that each picture in a set was of the same object changing over time. Young children's ability to comprehend the chronological order of the picture sets is supported by previous research demonstrating that by 3 years of age children understand and can construct three-picture sequences of familiar events (Fivush \& Mandler, 1985; Gelman, Bullock, \& Meck,1980). If, however, a child began to discuss an event in reverse order, the experimenter corrected the child by reminding him or her of which picture was first in the sequence.

Following the presentation of each picture set, the researcher asked each child three questions: (a) "What happened to the (object name)?" (b) "How did it (word used by the child)?” and (c) "Why did it (word used by child)?" For example, if a child answered "What happened to the duck?" by saying "It grew,", the experimenter would ask, "How did it grow?" and "Why did it grow?" If a child did not use a change-of-state verb in his or her description (e.g., "It's a baby

duck, then a kid duck, then a mommy duck."), the experimenter asked, "How did it change like that?" and "Why did it change like that?" This questioning strategy was designed not only to elicit a description of the event but also to encourage children to talk about the specific causal mechanisms responsible for the change.

\section{Coding}

Reference to growth. Using transcripts of the sessions, two coders independently coded all uses of the word grow (or variations) to determine whether the term was actually being 
used to describe the depicted event. As in Study 1, we focused exclusively on instances in which children were judged to describe the depicted event as a growth event (e.g., "[The sponge] growed and growed"), as opposed to talking about unpictured aspects of the event occurring either before or after the depicted change (e.g., "[The flower] just grew then it got dead"), or talking about the event in reverse order (e.g., "[The fish] was a baby then grew up to be a daddy") Agreement for determining which utterances constituted a description of the event was $90 \%$. Disagreements tended to be due to one coder missing an aspect of the utterance, such as when one coder judged a child to be describing the flower as growing when the other coder noted that the child seemed to be explaining the picture sets in reverse order. All disagreements were resolved through discussion.

Explanations. Children's explanations of picture sets were coded to investigate whether children explained events from different domains in distinct ways. The coding categories for Study 2 were identical to those described in Study 1: organic cause, human cause, other, and no explanation. Intercoder reliability was established by two coders independently coding $25 \%$ of the transcripts. Using Cohen's kappa as the agreement statistic, reliability for this decision was .77 (percent agreement $=81 \%$ ), with all disagreements resolved through discussion.

\section{Results and Discussion}

\section{Children's Reference to Growth}

A parallel research question to the first one explored in Study 1 asks whether children mainly referred to increases in size of biological items as growth or whether they described a variety of changes in terms of growth. It is important to emphasize that when children simply describe a change as a growth event this does not necessarily tell us whether they have an understanding of the specific processes involved in biological growth. This analysis does, 
however, help us to identify whether children differentiate by domain in terms of the events they are willing to describe as growth. If children's understanding of growth is indeed a core component of children's biological theory (as has been suggested in the literature; e.g., Inagaki \& Hatano, 1996), it is necessary to examine the situations for which children spontaneously invoke this concept.

In contrast to mothers who only talked about increases in size as growth, children in this study talked about both increasing and decreasing events as growth. As a result, two sets of analysis were necessary. The first analysis investigated children's descriptions of the increase and decrease in size of biological and artifact items. The second analysis examined children's descriptions of the increase in size of biological, artifact, and natural-kind items.

Biological and artifact items. A 2 (age: 3 years, 5 years) x 2 (domain: biological, artifact) x 2 (direction: increasing, decreasing) mixed ANOVA was conducted on the number of picture sets that children described in terms of growth. In this ANOVA, age was a betweensubjects factor, and domain and direction were within-subjects factors. The mean number of picture sets (by event type) that 3- and 5-year-old children identified as growth is shown in the first two columns of Table 4. Note that children's means are based on three items of each type, whereas in Study 1 mothers only saw two items of each type.

As in earlier analyses, inspection of the means and standard deviations raised concerns about constrained variance. Therefore, the ANOVA was repeated with the data transformed using a natural $\log$ arithmic transformation $\left[\ln \left(\begin{array}{ll}1 & 1 \mathrm{dv}\end{array}\right)\right]$ to stabilize spread. The results reported are those from the original ANOVA that were replicated by the analysis using transformed data.

The ANOVA showed significant main effects in the predicted directions for domain and direction, $\mathrm{F}(1,30)=31.84, \mathrm{p}<.001$ and $\mathrm{F}(1,30)=79.32, \mathrm{p}<.001$, respectively. In addition, the Direction $x$ Domain interaction was significant, $F(1,30)=31.88, \mathrm{p}<.001$. Tukey's HSD post hoc 
analysis of this interaction indicates that children referred to growth more for events in which a biological object increased in size than for any other type of event, $\mathrm{p}<.05$.

[Insert Table 4]

To explore further the kinds of changes that children might be willing to describe as growth, we next look at children's reasoning about three additional events depicting the increase in size of nonliving natural-kind objects (a popcorn kernel popping, the waxing of the moon, and crystal accretion). These items are interesting because they are similar to biological items in that they are natural, yet they are also similar to human-made artifacts in that they are not alive. In addition, the mechanisms of change are likely to be unfamiliar to children. Investigating the ways children label these events, then, may provide additional insight into the kinds of events children are willing to describe as growth.

Biological, artifact, and natural-kind items. The significance of the difference among children's talk about growth for biological, artifact, and natural-kind increasing events was compared in a 2 (age: 3 years, 5 years) x 3 (domain: biological, artifact, natural kind) mixed ANOVA on the number of picture sets per event type which children referred to as growth. Because the Box's $\mathrm{M}$ test for homogeneity of variance was significant, $\mathrm{F}(6,6520)=2.23, \mathrm{p}=.037$, we conducted a second analysis using data transformed logarithmically to stabilize spread (as described earlier). The Box's M test from the analysis using transformed data was not significant ( $\mathrm{p}=.08$ ), suggesting that the transformation successfully stabilized the spread. The transformed data wholly supported the findings from the original ANOVA, which are reported next.

Results indicate an overall effect of domain, $F(2,29)=22.61, p<.001$, with post hoc analyses showing that children referred to growth more for biological events than for either artifact or natural-kind events (HSD, $\mathrm{p}<.05)$. Unlike mothers, children did not differentiate between artifact and natural-kind events in their reference to growth. No effects of age were indicated by the analysis. 
Despite children's general domain-specific tendency, there were a number of instances in which children labeled nonbiological events as growth. In most instances children used grow in nonbiological contexts without any indication that this might be unusual (e.g., The sponge "grew"). At other times, children were more specific about their application of the term (e.g., "[The moon] kept growing like people grow" or "[The building] grew, just like plants!"). A few children, however, seemed to be struggling with the boundary between things that increase in size by "growing" and things that increase in size for other reasons. For example, one 3-year-old child said of the crystal, "They kept growing bigger and bigger. ... I don't really know how crystals might grow. I really don't know. It's so funny to me that crystals can grow." In this example, the child seems to appreciate that crystals may not be among the kinds of things that grow, yet is unable to think of an alternative way to describe the event. Unlike mothers in Study 1, however, none of the children in this study clearly qualified their use of grow for nonbiological events.

The preceding examples suggest that children vary in whether they think of growth as a specifically biological event. To better assess what children understand about the relationship between growth and biological kinds, it is necessary to look more closely at the explanations they provide for events occurring in different domains. For example, although some children might be willing to talk about nonbiological events as growth, they might not explain these events in terms of biological causal mechanisms. The following example illustrates this point nicely:

Experimenter: Can you tell me what happened to the sponge?

Child: $\quad$ It grew.

Experimenter: How did it grow?

Child: I don't know how sponges grow 
but I'll tell you how I think they grow. I think they just got pictures of them farther away. This one was so far away; this one was kinda, teeny bit close up; this one was much close up.

Even though this 5-year-old child describes the event as growth, her explanation is clearly non-biological. The next section systematically examines the causal mechanisms children used to explain the depicted changes.

\section{Children's Explanations}

Biological and artifact items. In addition to differentiating by domain in the events children described as growth, we asked whether children also differentiated by domain in the causal mechanisms they used to explain changes in different domains. Patterns in children's use of the three explanation categories (organic cause, human cause, other) were investigated using data analysis techniques similar to those described in Study 1. Analyses were based on the number of picture sets per event type (biological increases, biological decreases, artifact increases, and artifact decreases) for which children used each of the explanation categories to describe the depicted change. All causal explanation types were considered to be dependent measures with scores ranging from 0 (child did not use the given explanation to describe any of the picture sets within a category) to 3 (child described all of the picture sets within a category with that explanation). These scores were submitted to a multivariate analysis of variance in which age was a between-subjects factor and explanation type, domain, and direction were withinsubjects factors.

Results of this analysis show a significant Box's M test for homogeneity of variance, $\mathrm{F}(78$, 2,842) 5 1.35, p 5.022; therefore, we conducted a second analysis using the logarithmic data transformation described for previous analyses $\left[\ln \left(\begin{array}{ll}1 & 1 \mathrm{dv})\end{array}\right]\right.$. Transforming the data successfully 
stabilized spread, as evidence by a nonsignificant result for the Box's M test (p 5.10). Unless noted, the transformed data supported the findings from the original analysis, which are reported next.

The analysis revealed a multivariate effect of age such that 5-year-old children offered more explanations than did 3-year-old children, $\mathrm{F}(1,30) 512.44, \mathrm{p} 5.001$. On average, 5-year-old children explained 10.81 events whereas younger children explained 6.88 events (of 12). A main effect of explanation type was also found, with children offering human cause explanations on more picture sets (M 5 3.91) than either organic cause (M 5 2.22) or other explanations (M 5 2.72), $\mathrm{p}<.05$, by Tukey's HSD post hoc test. In addition, the analysis revealed several significant multivariate interactions. The following two-way interactions were significant: Age x Domain (significant in the analysis using transformed data only), $\mathrm{F}(1,30) 5$ 5.28, $\mathrm{p}<.05$; Explanation Type x Domain, F(2,29) 5 40.90, p <.001; Explanation Type x Direction, $\mathrm{F}(2,29) 511.77, \mathrm{p}<.001$; and Domain $x$ Direction, $\mathrm{F}(1,30) 58.72, \mathrm{p}<.01$. These interactions were not analyzed further as they are qualified by the significant three-way interactions of which they are a part.

First, the analysis revealed an interaction among explanation type, domain, and direction, $\mathrm{F}(2,29) 57.20, \mathrm{p}<.01$ (see Table 5 for means). Post hoc analysis of this interaction revealed that when explaining biological events, children used organic cause explanation to explain more increasing than decreasing events (HSD, $\mathrm{p}<.05$ ). They did not differentiate by direction in their use of human cause or other explanations. When explaining the change in size of artifacts, children's use of each explanation type was equal for increasing and decreasing events.

The MANOVA also revealed an Age x Explanation Type x Domain interaction, F(2, 29) $54.23, \mathrm{p}<.05$. Tukey's HSD post hoc analyses indicate that although 3 -year-old children demonstrated equal use of the three explanation types when explaining biological events, they explained more artifact events with human cause and other explanations than with organic 
cause explanations, $\mathrm{p}<.05$ (see Figure 1). In contrast to 3-year-olds, 5-year-old children emphasized organic cause and other explanations over human cause explanations when describing biological events. In addition, 5-year-old children used human cause explanations more than either biological or other explanations when explaining artifact events, $\mathrm{p}<.05$ (see Figure 2).

Natural-kind items. This section compares children's explanations of biological and artifact increases to the causes they used to explain natural-kind increases in size (moon, crystal, popcorn). In contrast to mothers in Study 1, children often used biological, human cause, and other explanations to explain natural-kind events, explaining $65 \%$ of the natural-kind events with at least one type of explanation (mothers only explained $12.5 \%$ of natural-kind events). In the following analysis, we explored whether children explained natural-kind events with causal mechanisms usually associated with biological growth (e.g., organic cause), with causes associated with artifact change (e.g., human cause), or in some other way.

[Insert Table 5]

[Insert Figure 1 and 2]

Children's explanations of natural-kind events were compared with their explanations of biological and artifact increases with an Age x Explanation Type x Domain MANOVA. Analyses were based on the number of picture sets for which children used each of the explanation categories to describe the depicted changes. Thus, each child received three scores (ranging from 0 to 3 ) for each of the three types of picture set (biological increase, artifact increase, natural-kind increase).

A main effect of age revealed that 5-year-old children explained more events than did 3year-old children, $\mathrm{F}(1,30)=15.55, \mathrm{p}<.001$. In addition, explanation type and domain interacted, $\mathrm{F}(4,27)=27.08, \mathrm{p}<.001$, as did age, explanation type, and domain, $\mathrm{F}(4,27)=5.29, \mathrm{p}<.001$. Post hoc analysis of the three-way interaction revealed results that replicated the patterns of findings for biological and artifact events indicated in the previous analysis. With regard to 
the question of how children explained natural-kind events, this analysis indicated that neither 3-year-old nor 5-year-old children emphasized any of the three explanation types when explaining natural-kind events.

\section{Summary of Study 2 Findings}

At the onset of this work we reasoned that preschool children might initially understand growth to refer generally to increases in size, and later narrow down their concept of growth to include only biological increases. The results presented here, however, suggest that children in this study did not interpret growth as simply meaning "gets bigger." Instead, they primarily identified events in which biological objects increased in size as growth. This set of findings extends previous work in that children were shown to exhibit evidence of domain specificity even when asked to reason about events in which nonbiological objects actually get bigger, rather than about impossible increases (as in earlier studies). There were, however, indications that children at both ages were still refining their understandings. Specifically, both 3- and 5-year-old children sometimes described nonbiological increasing events in terms of growth.

Investigation of children's explanations of events supports the idea that children also begin to develop domain-specific causal understandings by the preschool years, and that these understandings are refined over time. Although children at both ages emphasized human cause explanations over organic cause explanations for artifact events, only 5-yearold children also emphasized organic cause explanations over human cause explanations for biological events. Children in both age groups used all of the explanation types equally for natural-kind events, perhaps suggesting that they were unsure of the most appropriate ways to explain events in this domain.

\section{General Discussion}


In these studies, we investigated young children's understanding of events in which biological and nonbiological objects change size, and how parents may help children learn to distinguish by domain in their reasoning about such events. Findings from these studies suggest that by the preschool years, children already know a great deal about biological and nonbiological change-in-size events. Furthermore, mothers may play a role in guiding children's understanding of domain differences. Next, we discuss in greater depth the implications of our findings, with an emphasis on highlighting both consistencies and inconsistencies in the ways children and mothers talked about events.

Understanding of Growth as a Biological Event

In Study 1, our interest was in asking whether mothers distinguish between biological and non-biological events in their conversations with children. One of our goals was to examine which events mothers labeled as growth, as this concept may be central to children's developing understanding of biology as an autonomous domain (Inagaki \& Hatano, 1996). This is a meaningful research question as patterns in adult speech to children might influence children's conceptual understandings (see Gopnik \& Meltzoff, 1997). Results indicated that although mothers concentrated their talk about growth on biological increases in size, they also occasionally talked about nonbiological events in terms of growth. Mothers' references to growth in nonbiological contexts, however, were consistent with social conventions regarding use of the word grow.

That mothers in this study occasionally used growth outside of the biological context highlights the fact that natural language is often imprecise and ambiguous, resting on social conventions rather than semantic facts. If, as we argue, mother-child conversations are an important context for the negotiation of theory-relevant understandings, it becomes important to consider the impact of non-biological uses of grow on children's understandings. On 
the one hand, this kind of flexible use could confuse children as they negotiate the connection between their theories of biology and the concept of growth. On the other hand, children may attend primarily to what is most consistent in mother talk (i.e., that growth applies to biological events) and may disregard other (nonbiological) uses. It is important to point out that children do not always have to solve this puzzle on their own. Some mothers in this study included information in their language that may have helped their children interpret nonbiological references to growth, for instance, by explaining how their use of grow for the crystal differs from more typical uses. Such conversations, although admittedly infrequent in our data, may be contexts that are particularly helpful to children as they begin to recognize the conflict between apparent growth and actual biological growth.

Study 2 results indicated that, like mothers, children focused their use of grow on biological events in which an object increased in size. Also like mothers, children occasionally described nonbiological events as growth. The contexts within which mothers and children referenced grow, however, differed. Mothers' reference to growth in nonbiological contexts could be seen as consistent with social conventions (i.e., primarily restricted to the crystal), whereas children talked about a variety of events as growth, including biological and artifact increases and decreases, and natural-kind increases.

It is unclear what these nonbiological uses of grow indicate about conceptual understandings. One possibility is that speakers who use grow outside of the biological realm have an incomplete understanding of the domain-specific nature of growth. A second possibility is that they are confused about the ontology of specific items (e.g., they believe that crystals are alive). Finally, some speakers who talked about nonbiological events in terms of growth may be aware that growth is a biological event and may know which items are biological. Such speakers may be demonstrating an appreciation for the multiple meanings of growth apparent in 
everyday language use. We suspect that mothers fall into the latter category, whereas children fall into one of the two previous categories.

One approach to considering these possibilities is to look more closely at how grow is used outside of the biological realm. For instance, do particular items elicit descriptions that reference growth? This was certainly the case in Study 1. Of the 18 cases in which mothers used grow to describe a nonbiological event, 14 were for the crystal. This finding is interesting in that crystal accretion is often called growth by both lay people and scientists and "crystalgrowing kits" are found in many toy stores. Thus, mothers' use of grow for this event may be a reflection of accepted word usage and not necessarily an indication that they are unaware that growth is most commonly used to describe a specific biological event (nor is it likely to be an indication that they believe crystals to be alive). In Study 2, however, children talked about growth for a much wider variety of events, suggesting that their understanding of the concept is broader than that of mothers and may not be based on an understanding of conventional word use.

The current data do not allow us to establish whether children's flexible use of grow is due to ontological confusion or an incomplete understanding that grow is predominantly used to refer to biological increases in size. However, it is likely that, even within a single age group, individual differences will be apparent in children's conceptual understandings. For example, in our data we found that some individuals adhered to strict domain distinctions, whereas others were more fluid in their use of grow. Specifically, two 3-year-old children and one 5-year-old child identified all three biological events as growth (and none of the other events). The majority of children, however, demonstrated variable patterns in their use of grow. Longitudinal work that tracks the progression of individual children's understanding of theory-laden concepts would be helpful in attempts to clarify the likely source of domain blurring. It would not be surprising to see such an investigation reveal a nonlinear relationship between age and children's reference to 
growth for nonbiological events (possibly a U-shaped curve). Future researchers might also wish to collect data on children's ontological distinctions.

Overall, however, the findings from these studies suggest that both mothers and children appreciate that the most common use of the word grow is to describe biological increases in size. This result is consistent with the Rosengren et al. (1991) finding that by 3 years of age children differentiated between animals and artifacts in their decisions about the possibility for growth (see also Backsheider et al., 1993). In addition to supporting earlier findings, the current study enhances our understanding of children's reasoning about growth by showing that children differentiate by domain even when confronted with a wide variety of actual changes. In contrast to earlier work, children in this study were asked to discuss possible artifact increases (e.g., a beachball being blown up) rather than impossible artifact increases (e.g., a chair getting larger). Second, children were asked to discuss the increase in size of nonliving natural kinds. This last event type was included to explore whether children considered growth to be a specifically biological event or whether they might consider it to be a natural event that appropriately describes the increase in size of both living and nonliving natural kinds. Therefore, our study required children to make judgments about real-world events with similar perceptual appearances (but different causes) that occurred in several domains. Even when this additional layer of complexity was added, children were remarkably domain specific in their use of grow to describe events.

The conclusion that children recognize growth to be a primarily biological event is further reinforced by several studies that have reached the same conclusion with varying methodologies. Previous work has examined children's reasoning about changes occurring in a variety of domains through the use of forced-choice tasks. Although forced-choice tasks tend to be more carefully controlled, they may not thoroughly reveal how children themselves think about events. In the current study, we used an open-ended interview to 
explore children's descriptions and explanations of change. In allowing children to be unconstrained in their description of events, we found that they often mentioned growth and did so primarily for events in which a biological object increased in size. Thus, by replicating previous findings with a converging methodology, we can more confidently claim that preschool children recognize growth to be a domain-specific process in which a biological object increases in size.

\section{Knowledge of Domain-Specific Causal Mechanisms}

Data on patterns of word use alone may be insufficient to evaluate the nature of one's understandings. As Keil and Silberstein (1996) have argued, "We can't fully characterize how concepts are represented without also characterizing the larger systems of explanation in which they are embedded" (p. 630). Thus, in addition to examining mothers' and children's mentions of growth, a second line of inquiry examined the causal mechanisms used to explain change. Because we predicted that mother- child conversations might be a context for children's developing understanding of biology as a distinct domain, we looked for evidence that mothers modeled the use of domain-specific causal mechanisms. We then explored whether children differentiated by domain in the ways they explained events.

Our findings indicate that mothers clearly favored specific causal mechanisms for events occurring in different domains. They explained artifact events with human cause explanations, and biological events with organic cause explanations. Neither explanation type was used to explain natural-kind events, which often went unexplained. These findings are particularly interesting as, as Coley (2000) pointed out, there is currently very little research available on adults' conceptions of biological and nonbiological events. Although not specifically aimed at responding to this deficit, these findings advance our knowledge in the area. 
In our analysis of the Study 2 data, we found evidence for a developmental influence on children's conceptual understandings. In particular, whereas 5- year-old children demonstrated causal understandings similar to that of mothers, 3-year-old children did not demonstrate domain-specific causal reasoning to the same extent. Instead, 3-year-olds evidenced domainspecific causal understandings for artifact events only, explaining these events primarily with human cause explanations. For biological events, 3-year-old children used biological and human cause explanations equally. Children at both ages seemed unsure of appropriate explanations for natural-kind events, using biological, human cause, and other explanations equally. In contrast to mothers, though, children often made an attempt to explain these events, explaining $65 \%$ of events in this domain (as compared with $13 \%$ for mothers).

That even 3-year-old children had begun to make domain distinctions indicates an early appreciation that events occurring in different domains are likely to be due to different causes. For instance, children's early understanding of domain specificity in explanatory frameworks is illustrated in an example in which a 3-year-old child described the increase in size of the moon by saying, "It grew big." When asked how that happened, the child replied, "The food," and then went on to laugh and say, "I'm funny." The child in this example seems to understand that food is important for growth, yet recognizes the humor in trying to apply this knowledge to his assertion that the moon grew. Incidentally, this example also demonstrates that when given opportunities to talk about events in a spontaneous way, children may reveal aspects of their understandings that researchers might not think to ask about in more structured paradigms.

In conclusion, in children's everyday lives they are likely to encounter a variety of events in which an object changes in size. In addition, children are likely to consider these events in the company of others. In the present studies, we aimed to increase our understanding of how children reason about diverse events, and whether conversations with mothers may 
contribute to children's understandings. Our findings support and extend current work suggesting that children construct theories that divide the world into distinct domains with distinct causal mechanisms. Furthermore, beyond simply documenting children's understandings, this work begins to explore one context in which children may have opportunities to develop their understandings. Although mothers in this study did not offer elaborate explanations of all events, they did explain many events and did so in domain-specific ways. Our results are similar to those of Gelman et al. (1998) in that mothers did not always take advantage of opportunities to explain events to their children. We agree with Gelman et al. in arguing that there are several subtle things that mothers do that may, in fact, be informative for children (e.g., domain-specific application of growth, discussion of ambiguous cases). Thus, the results of this work encourage us to keep open the possibility that mother-child conversation may be an important source of information for children as they negotiate their developing theories of the world. This finding provides a foundation for future work investigating whether and how children use the information provided by mothers about everyday events.

\section{References}

Backsheider, A., Shatz, M., \& Gelman, S. (1993). Preschoolers' ability to distinguish living kinds as a function of regrowth. Child Development, 64, 1242-1257.

Bakeman, R., \& Gottman, J. M. (1997). Observing interaction: An introduction to sequential analysis (2nd ed.). New York: Cambridge University Press.

Bartsch, K., \& Wellman, H. (1995). Children talk about the mind. New York: Oxford University Press.

Beals, D. E. (1993). Explanatory talk in low-income families' mealtime conversations. Applied Psycholinguistics, 14, 489-513. 
Carey, S., \& Spelke, E. (1994). Domain-specific knowledge and conceptual change. In L. A.

Hirschfeld \& S. A. Gelman (Eds.), Mapping the mind: Domain specificity in cognition and culture (pp. 169-200). New York: Cambridge University Press.

Coley, J. D. (2000). On the importance of comparative research: The case of folkbiology. Child Development, 71, 82-90.

Crowley, K., Callanan, M. A., Jipson, J. L., Galco, J., Topping, K., \& Shrager, J. (2001).

Shared scientific thinking in everyday parent-child activity. Science Education, 85, 712-732.

Emerson, J. D., \& Soto, M. A. (1983). Transforming data. In D. C. Hoaglin, F. Mosteller, \& J. W. Tukey (Eds.), Understanding robust and exploratory data analysis (pp. 97-128). New York: Wiley.

Fivush, R., \& Mandler, J. M. (1985). Developmental changes in the understanding of temporal sequence. Child Development, 56, 1437-1446.

Gauvain, M. (2001). The social context of cognitive development. New York: Guilford.

Gelman, R., Bullock, M., \& Meck, E. (1980). Preschoolers’ understanding of simple object transformations. Child Development, 51, 691-699.

Gelman, S., \& Coley, J. (1991). Language and categorization: The acquisition of natural kind terms. In S. A. Gelman \& J. P. Byrnes (Eds.), Perspectives on language and thought (pp. 146-196). Cambridge, England: Cambridge University Press.

Gelman, S. A., Coley, J. D., Rosengren, K. S., Hartman, E., \& Pappas, A. (1998). Beyond labeling: The role of maternal input in the acquisition of richly structured categories. Monographs of the Society for Research in Child Development, 63 (1) Serial No. 256, 1-148.

Gelman, S. A., \& Gottfried, G. M. (1996). Children's causal explanations for animate and inanimate motion. Child Development, 67, 1970-1987.

Gelman, S. A., \& Kremer, K. E. (1991). Understadning natural cause: Children's explanations of how objects

and their properties originate. Child Development, 62, 396-414. 
Gopnik, A., \& Meltzoff, A. N. (1997). Words, thoughts, and theories. Cambridge, MA: MIT Press.

Gopnik, A., \& Wellman, H. M. (1994). The theory theory. In L. A. Hirschfeld \& S. A. Gelman (Eds.), Mapping the mind: Domain specificity in cognition and culture (pp. 257-293). New York: Cambridge University Press.

Hatano, G., \& Inagaki, K. (1994). Young children's naive theory of biology. Cognition, 50, 171188.

Hickling, A. K., \& Gelman, S. (1995). How does your garden grow? Early conceptualization of seeds and their place in the plant growth cycle. Child Development, 66, 856-876.

Inagaki, K., \& Hatano, G. (1987). Young children's spontaneous personification as analogy. Child Development, 58, 1013-1020.

Inagaki, K., \& Hatano, G. (1996). Young children's recognition of commonalities between animals and plants. Child Development, 67, 2823-2840.

Keil, F. C. (1994). The birth and nurturance of concepts by domains: The origins of concepts of living things. In L. A. Hirschfeld \& S. A. Gelman (Eds.), Mapping the mind: Domain specificity in cognition and culture (pp. 234-254). New York: Cambridge University Press.

Keil, F. C., \& Silberstein, C. S. (1996). Schooling and the acquisition of theoretical knowledge. In D. R. Olson \& N. Torrance (Eds.), The handbook of education and human development: New models of learning, teaching and schooling (pp. 621-645). Oxford, England: Blackwell.

Rogoff, B. (1990). Apprenticeship in thinking: Cognitive development in social context. New York: Oxford University Press.

Rosengren, K. S., Gelman, S. A., Kalish, C. W., \& McCormick, M. (1991). As time goes by: Children's early understanding of growth in animals. Child Development, 62, 1302-1320.

Ruffman, T., Slade, L., \& Crowe, E. (2002). The relation between children's and mothers' mental state language and theory of mind understanding. Child Development, 73, 734-751. 
Sabbagh, M. A., \& Callanan, M. A. (1998). Metarepresentation in action: 3-, 4-, and 5-year-olds' developing theories of mind in parent-child conversations. Developmental Psychology, 34, 491502.

Snow, C. E., \& Kurland, B. F. (1996). Sticking to the point: Talk about magnets as a preparation for literacy. In D. Hicks (Ed.), Child discourse and social learning. An interdisciplinary perspective (pp. 189-220). New York: Cambridge University Press.

Springer, K., \& Keil, F. (1991). Early differentiation of causal mechanisms appropriate to biological and non-biological kinds. Child Development, 62, 767-781.

Wellman, H. M., \& Gelman, S. A. (1998). Knowledge acquisition in foundational domains. In D. Kuhn \& R. Siegler (Eds.), Cognition, perception, and language: Vol. 2. Handbook of Child Psychology (5th ed., pp. 523-574). New York: Wiley.

Table 1

Items

\begin{tabular}{llll}
\hline & & \multicolumn{1}{c}{ Domain } & \\
\cline { 2 - 4 } Age & \multicolumn{1}{c}{ Biological } & \multicolumn{1}{c}{ Artifact } & Natural \\
\hline Increasing & Duck growing & Beachball expanding & Crystal accretion \\
& Mushroom growing & Sponge expanding & Moon waxing \\
& Baby growing $^{\mathbf{a}}$ & Building being erected & Popcorn popping \\
Decreasing & Puffer fish deflating $^{\mathbf{a}}$ & Balloon deflating & N/A \\
& Strawberry rotting & Pencil being sharpened & N/A \\
& Flower wilting $^{\mathrm{a}}$ & Soda Can being crushed & N/A \\
\hline
\end{tabular}

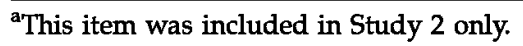

Table 2

Mothers' Mean (and Standard Deviation) Uses of the Word Grow for Increasing Events as a Function of Domain

\begin{tabular}{llcr}
\hline & \multicolumn{3}{c}{ Domain of change } \\
\cline { 2 - 4 } Age & Biological & Artifact & Natural \\
\hline 3-year-olds & $1.28(.58)$ & $.06(.24)$ & $.33(.49)$ \\
$4 \frac{1}{2}$ year-olds & $1.00(.59)$ & $.06(.24)$ & $.56(.71)$ \\
\hline
\end{tabular}

Note. Means are of two possible. 
Table3

Mothers' Mean (and Standard Deviation) Uses of Each Causal Mechanism as a Function of Direction and Domain

\begin{tabular}{lccr}
\hline & \multicolumn{3}{c}{ Explanation type } \\
\cline { 2 - 4 } Type of change & Organic cause & Human cause & Other \\
\hline Biological & & & \\
Increases & $.47(.56)$ & $.03(.17)$ & $.25(.44)$ \\
Decreases & $.19(.40)$ & $.19(.40)$ & $.75(.65)$ \\
Artifact & & & \\
Increases & $.69(.47)$ & $.75(.69)$ & $.67(.59)$ \\
Decreases & $.03(.17)$ & $.75(.50)$ & $1.08(.60)$ \\
\hline
\end{tabular}

Table 4

Children's Mean (and Standard Deviation) Uses of the Word Grow as a Function of Direction and Domain

\begin{tabular}{lcccc}
\hline & & \multicolumn{3}{c}{ Domain of change } \\
\cline { 3 - 5 } Direction of change & Age & Biological & Artifact & Natural \\
\hline Increasing & 3-year-olds & $1.43(1.09)$ & $.56(.96)$ & $.50(.73)$ \\
& 5-year-olds & $2.31(1.14)$ & $.38(.50)$ & $.88(.81)$ \\
Decreasing & 3-year-olds & $.25(.45)$ & $.19(.54)$ & $\mathrm{N} / \mathrm{A}$ \\
& 5-year-olds & $.19(.54)$ & $.00(.00)$ & $\mathrm{N} / \mathrm{A}$ \\
\hline
\end{tabular}

Note. Means are of three possible.

Table5

Children's Mean (and Standard Deviation) Uses of Each Causal Mechanism as a Function of Direction and Domain

\begin{tabular}{lccc}
\hline & \multicolumn{3}{c}{ Explanation type } \\
\cline { 2 - 4 } Type of change & Organic cause & Human cause & Other \\
\hline Biological & & & \\
Increases & $1.41(1.24)$ & $.25(44)$ & $.75(1.02)$ \\
Decreases & $.53(.84)$ & $.53(.67)$ & $.63(.61)$ \\
Artifact & & & $.44(.50)$ \\
Increases & $.22(.42)$ & $1.56(.95)$ & $.91(.82)$ \\
Decrease & $.06(.25)$ & $1.56(.95)$ & \\
\hline
\end{tabular}




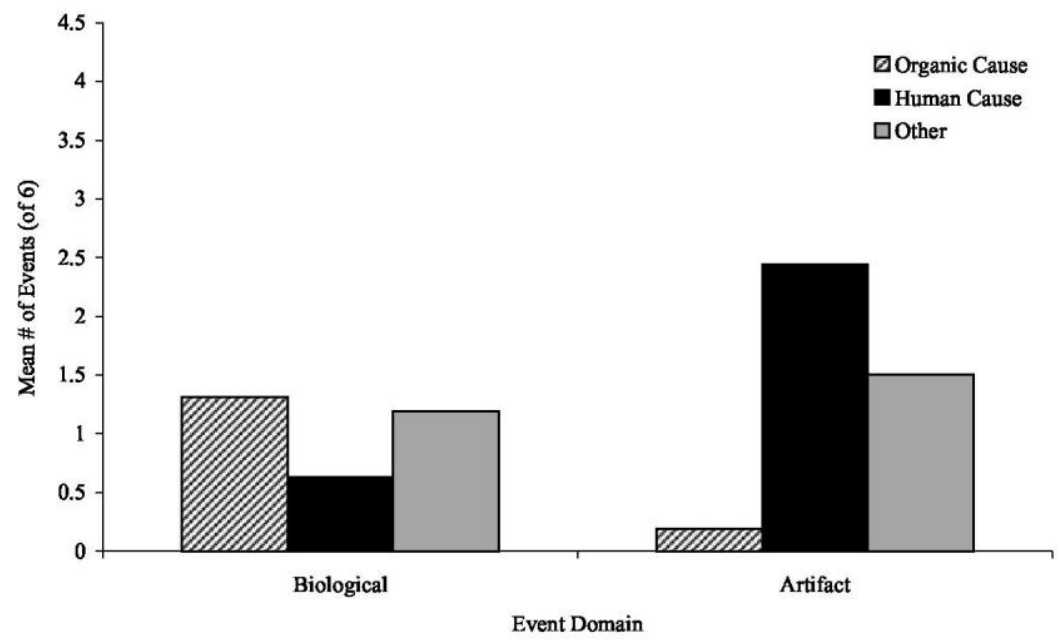

Figure 1. Three-year-old children's explanations of biological and artifact events.

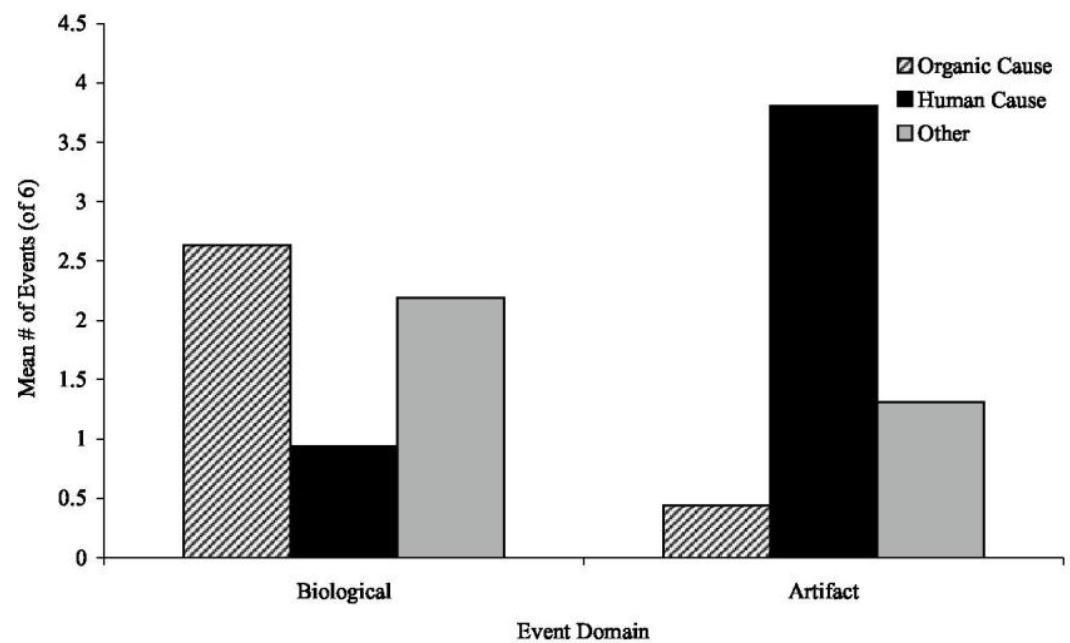

Figure 2. Five-year-old children's explanations of biological and artifact events. 